\title{
¿Son invisibles las mujeres científicas? ${ }^{1}$
}

\author{
María Teresa GARCíA NiETO \\ Universidad Complutense de Madrid \\ xyz@ccinf.ucm.es
}

Recibido: $18 / 10 / 2012$

Aceptado: 23/01/2013

\section{Resumen}

El Proyecto de Investigación para el Fomento de la Igualdad de Género en la Información Científica, subvencionado por el Instituto de la Mujer, del Ministerio de Sanidad, Política Social e Igualdad, constituye una investigación aplicada, destinada a desvelar la situación de invisibilidad de las mujeres científicas en la prensa española. El presente artículo describe las conclusiones del estudio realizado sobre la notoriedad de las mujeres investigadoras en la comunicación en Internet.

Palabras clave: Información científica, igualdad de género, comunicación y ciencia, comunicación y género, información y mujeres científicas.

\section{Are the Scientific Women Invisible?}

\begin{abstract}
The Research Project for the Promotion of Social Gender Equality in the Scientific Information, subsidized by The Institute of Women, in the Ministry of Health, Social Politics and Equality, constitutes an applied research, destined to reveal the situation of invisibility of the scientific women in the Spanish press. This article describes the conclusions of the study realized about the reputation of the investigative women in the communication in Internet.

Keywords: Scientific Information, Social Gender Equality, Communication and Science, Communication and Gender, Information and Scientific Women.

\section{Referencia normalizada}

GARCÍA NIETO, María Teresa (2013): “¿Son invisibles las mujeres científicas?”. Estudios sobre el mensaje periodístico. Vol. 19. Núm. especial abril, págs.: 783-792. Madrid, Servicio de Publicaciones de la Universidad Complutense.
\end{abstract}

Sumario: 1. Las providencias periodísticas. 2. Representación e imagen de las mujeres científicas: un camino, diversas sendas; 2.1. Las mujeres en la información científica. Análisis de los discursos periodísticos; 2.2. La percepción de la ciencia. ¿Y la imagen pública de las mujeres científicas?. 3. La notoriedad de las mujeres científicas en Internet. 4. Referencias bibliográficas.

\section{Las providencias periodísticas}

Maxwell McCombs y Donal L.Shaw, profesores de Periodismo de la Universidad de Carolina del Norte, en su estudio sobre la Teoría del Establecimiento de la Agenda de los Medios, publicado en 1972, afirmaron de forma incontestable que las decisiones de editores y redactores de los medios de comunicación condicionan indefectiblemente el interés, la notoriedad y la opinión de los públicos. La selección de contenidos y asuntos, la ubicación de la noticia en la página correspondiente, el espacio o el tiempo dedicado a cada una de ellas, constituyen las providencias determinantes del catálogo temático y actitudinal de los públicos.

El corpus teórico del periodismo, dos décadas más tarde, encontrará un nuevo pilar epistemológico, complemento adecuado a la mencionada propuesta, en la denomi-

1 Proyecto IFIGIC, Ref. 2011-004-INV-00016. Instituto de la Mujer 
nada Teoría de los Marcos Referenciales (frames), desarrollada por Robert M. Entman, profesor de medios y asuntos públicos en la School of Media and Public Affairs de la Universidad George Washington (GMU). No olvidemos que Entman fue el responsable de aplicar al ámbito del periodismo la Teoría del Encuadre en la organización de la experiencia, expuesta por Erwing Goffman en 1974. Los postulados de Goffman emanan del llamado "interaccionismo simbólico", (George Herbert Mead, 1934; Herbert Blumer, 1938; Peter Berger y Thomas Luchmann, 1968), en el marco de la sociología de los grupos pequeños y de la psicología social. Entman aplicará de forma novedosa este enfoque al análisis de las narraciones periodísticas, lo que el mismo llamará el estudio de los news frames.

El concepto de enmarcar, poner un marco interpretativo, consiste en seleccionar algunos aspectos de una realidad percibida y hacerlos más relevantes en el texto que se comunica, de tal modo que promueva una definición particular del problema, la interpretación causal, la evaluación moral, y/o la recomendación de su tratamiento desde un enfoque determinado. (1993, p. 53). Al redactar la noticia, el periodista enmarca el hecho, lo encuadra convenientemente y este marco interpretativo va acondicionar la percepción que los públicos puedan tener del acontecimiento comunicado.

Los ciudadanos y las ciudadanas construyen, en una gran medida, sus imágenes mentales como conjuntos de creencias forjadas a partir de las informaciones de los medios de comunicación. Sus gustos y preferencias, sus actitudes y opiniones, sus predisposiciones a favor o en contra, se fraguan como resultado del procesamiento de la información que reciben, entre muchas otras, en forma de mensajes periodísticos. Por este motivo, omitir la referencia a una persona, a un colectivo, a una organización, o a un acontecimiento, en la información periodística, produce como resultado su invisibilidad pública, su ocultación y en definitiva una carencia absoluta de notoriedad y de relevancia social.

Aunque la mayoría de las investigaciones periodísticas realizadas con estos parámetros teóricos han sido desarrolladas en el ámbito de la comunicación política, entendemos que sus postulados son también aplicables a la esfera de la ciencia y desde ellos, se formula y se justifica la hipótesis que hemos planteado en el trabajo de Investigación para el Fomento de la Igualdad de Género en la Información Científica.

\section{Representación e imagen de las mujeres científicas: un camino, diversas sen- das}

El Proyecto de investigación para el Fomento de la Igualdad de Genero en la información Científica (IFIGIC), financiado por el Instituto de la Mujer, del Ministerio de Sanidad, Política Social e Igualdad, parte de la hipótesis de la invisibilidad de las mujeres científicas en los medios de comunicación españoles. La muy parca presencia de las mujeres científicas españolas en las informaciones de ciencia, investigación y tecnología, publicadas en los medios de comunicación de nuestro país, presumiblemente acentúa el desinterés, refuerza el desconocimiento y crea una imagen imprecisa y desdibujada de este colectivo.

Se ha determinado identificar varias facetas en el desarrollo de la investigación, para favorecer una aproximación poliédrica al objeto de investigación, desde los im- 
prescindibles ángulos para su conocimiento. Por un lado se pretende conocer el grado de notoriedad y la representación mediática de las mujeres científicas en España; por otro, es preciso explorar su imagen pública o percepción social. Por último, resulta imprescindible identificar la realidad misma de las investigadoras a nivel nacional, tanto cualitativa y cuantitativamente. Con este planteamiento, podremos comprobar, a posteriori, el grado de correspondencia entre la representación mediática de las mujeres científicas, su imagen pública y su situación real en España.

En definitiva, nuestra investigación pretende cimentar una respuesta adecuada a lo establecido en la Ley de Economía Sostenible, en cuanto su propósito de lograr la promoción de la integración de la mujer en los diversos campos del saber científico y tecnológico; así como, el fomento de la igualdad entre las mujeres y los hombres de ciencia en los procesos de formación, investigación y también, en el ejercicio de su profesión. Vayamos por partes.

\subsection{Las mujeres en la información científica. Análisis de los discursos periodísticos} En primer lugar, planteamos la necesidad de evidenciar el grado de presencia y la notoriedad de las mujeres científicas en la comunicación pública española. Para ello hemos tomado como muestra de representación a la prensa escrita, periódicos diarios de información general de pago; asimismo hemos añadido, por su especial relevancia, lo publicado en Internet, en lengua española, por supuesto referido a la información científica.

La opción de considerar a la prensa diaria en papel, de pago, como el medio elegido para el análisis de los discursos periodísticos científicos, se debe a su mayor credibilidad como medio de divulgación científica. La televisión, pese a sus audiencias masivas, como fuente de información científica, es percibida por el público como un medio menos creíble, sólo por delante de las revistas semanales de información general y de la prensa gratuita, tal como muestra la V Encuesta de Percepción Social de la Ciencia 2010, realizada por la Fundación Española para la Ciencia y la Tecnología, última publicada al inicio de nuestra investigación.

La elección de las cinco cabeceras muestrales, todas de carácter generalista y de ámbito nacional, se hizo atendiendo a los criterios de mayor tirada y difusión. En un primer momento elegimos a los diarios El País, El Mundo, ABC, La Razón y Público. Pero, en el mes de febrero de 2012 sobrevino una circunstancia inesperada. El diario Público anunciaba la desaparición de su edición impresa, lo cual supuso la retirada de esta cabecera de la muestra de nuestra investigación. Tras ponderar diversas opciones, optamos por incorporar al diario La Vanguardia, un periódico histórico de la prensa española y catalana, por ser, en cuanto a su difusión, el tercer diario de información general de la prensa española de pago, según los datos elaborados por la OJD, Oficina para la Justificación de la Difusión, y por el EGM, Estudio General de Medios. Además, la incorporación de La Vanguardia permite realizar una primera comparación, en el tratamiento informativo sobre las mujeres científicas, tanto en la prensa de difusión nacional como en la de difusión autonómica y regional.

Tras conocer su grado de presencia y notoriedad, estudiamos la imagen de las mujeres científicas en las informaciones de los cinco diarios seleccionados. Para ello, 
como hemos dicho, tratamos de identificar las características, los prejuicios y los estereotipos de género en la representación mediática de las mujeres científicas, mediante el análisis de los diversos discursos periodísticos de estos rotativos.

En consonancia con las teorías antes citadas, respaldo epistemológico de nuestra investigación, las decisiones de los gestores del trabajo periodístico van a determinar, no sólo la visibilidad, la importancia y la notoriedad de las noticias, sino también su contenido y su enfoque, los cuales establecerán, en última instancia, la percepción social del hecho noticioso. Es muy curioso señalar como, según un estudio realizado por el diario El Mundo, publicado en su suplemento Yo Dona el tres de marzo de 2012, revela que solamente tres de cada diez artículos, en las diversas secciones de la prensa diaria, están firmados por mujeres, a excepción de las secciones de economía y deporte donde, lamentablemente, las mujeres están especialmente ausentes. Se trata, sin duda, de un fenómeno tremendamente significativo.

Por otro lado, se comprobó, según los datos del EGM, que el número de usuarios de Internet había experimentado un notable incremento durante el último año, alcanzando un alto grado de penetración de casi el sesenta por ciento de la población española. A la luz de estos pormenores, tomamos la decisión de realizar un estudio sobre la notoriedad de las mujeres y los hombres de ciencia, en la información en Internet. Esta disposición se confirmaría después muy eficaz a tenor de los resultados de la VI Encuesta de Percepción Social de la Ciencia 2012 de la FECYT, según los cuales la comunicación en Internet se sitúa por primera vez en el puesto de cabeza, como fuente de la información científica, por encima incluso del medio Televisión.

De esta manera, los respectivos análisis de las informaciones científicas publicadas en los diarios impresos de pago, antes citados, por un lado, y en Internet, por otro, nos aportan la posibilidad de comparar las circunstancias de ambos medios.

\subsection{La percepción de la ciencia. ¿Y la imagen pública de las mujeres científicas?}

Para conocer cuál es la percepción que la población española tiene de las mujeres científicas, en el momento actual, es necesario realizar una investigación de campo. Sin embargo, nuestro objetivo en este capítulo del estudio, es efectuar una primera aproximación para detectar las categorías y variables más significativas, determinantes de la imagen pública de las investigadoras españolas en función de su condición de mujeres. Se procura apreciar, si las hubiere, las posibles diferencias entre las opiniones que la población española, distinguiendo entre mujeres y hombres, tiene acerca de este colectivo. Se trata, por tanto, de un trabajo, que no pretende extraer conclusiones generalizables a toda la población, en términos cuantitativos; sino que trata de explorar, de realizar una prospección, que ofrezca una aproximación cualitativa al tema de nuestro interés.

Para lograr este propósito, planteamos la aplicación de la técnica del "grupo de discusión", implementándola con tres grupos de públicos diferenciados. Un grupo compuesto solamente por mujeres; otro formado por hombres y el tercero mixto, constituido por mujeres y hombres. Los criterios de selección de las personas que participaron en los grupos de discusión, profesionales en activo con formación superior universitaria, fueron acreditados posteriormente por dos trabajos muy relevantes. El primero es la in- 
vestigación internacional, titulada La Comprensión de la Ciencia, realizada por la Fundación BBVA, publicada el ocho de mayo de 2012. Se trata de un estudio comparado, realizado, en diez países de Europa y Estados Unidos, a mil quinientas personas, durante los meses de octubre y noviembre de 2011. La segunda, es la ya citada VI Encuesta de Percepción Social de la Ciencia de la FECYT, centrada en la población española, realizada mediante siete mil setecientas ochenta y cuatro entrevistas, en todo el territorio nacional, entre el veintiuno de febrero y el diez y ocho de abril de 2012.

Los dos estudios, sin duda alguna de una gran utilidad para nuestra investigación, ofrecen resultados muy reveladores y retratan, con gran exactitud, la percepción que los españoles y españolas tienen sobre la ciencia. Señalemos algunos de ellos. El interés de la población española sobre los temas científicos, similar al de la media europea, es de 5,7 en una escala de 0 a 10, próximo al de los temas internacionales, $5,6 \%$; y por encima del interés por los asuntos políticos, $4,5 \%$. Pese a todo, el interés por la ciencia se sitúa por debajo del manifestado para otras cuestiones, como la salud, el 7,9\%; el medio ambiente y la economía, el 6,7\%. No obstante, este índice ha crecido un $19 \%$ en los dos últimos años, situandose en un $15,5 \%$ en 2012 . Como dato negativo, señalamos que un gran porcentaje de la población, un $30 \%$, no tiene ningún interés por la ciencia y, además, uno de cada cuatro españoles afirma no entenderla. Por otra parte, la información que los encuestados declaran tener sobre la ciencia es más baja que la media europea, un 4,7\%. Un 18\% de los españoles dice leer noticias y suplementos en los periódicos, sobre temas de ciencia y tecnología, frente al $32 \%$ de la media europea.

Internet se ha convertido en la primera fuente de la información científica en España. Los datos no dejan lugar a duda. Internet representa el $41 \%$ de estas informaciones, seguida por la televisión, con un $31 \%$, y a distancia de la prensa diaria, con un $7,6 \%$. En cuanto a la confianza inspirada por las informaciones científicas de los diferentes soportes de divulgación científica y técnica, el primer lugar lo ocupan las revistas especializadas, con una puntuación del 4,2, en una escala de 1 a 5, seguidas de Internet, con un 3,56. Las informaciones de la televisión han visto incrementada su credibilidad en los últimos años, superando incluso a la prensa diaria, pasando de un 3,35 a un 3,5. Este interesante resultado nos da pie para plantear nuestras futuras investigaciones centradas en los mensajes televisivos y audiovisuales de la ciencia.

El nivel de conocimiento científico de la población española es muy bajo, un pobre $25 \%$, el mismo que Italia y Polonia y la mitad que el de Dinamarca, Países Bajos y Alemania. Como un dato preocupante, señalamos que el $46 \%$ de los españoles no puede ofrecer nombre alguno de un científico destacado. Además, la población encuestada no desarrolla muchas actividades para obtener informaciones y conocimientos científicos. Tan solo un 6\% asiste a charlas y conferencias sobre temas de ciencia. En Europa y Estados Unidos, la media de este parámetro se multiplica por dos. Es más, el $42 \%$ de los españoles confiesa tener una baja o muy baja educación científica, y solo menos del $12 \%$ piensa que su nivel de formación en asuntos de la ciencia es alto o muy alto.

La vinculación de los españoles y españolas con la ciencia es menor que en Europa. El $22 \%$ de la población conoce alguna persona dedicada a la ciencia; el $17 \%$ tiene amistad con algún científico o científica y el 16\% ha pensado, alguna vez, dedicarse 
a trabajos científicos. El perfil de las personas con mayor vinculación científica es el de los hombres, adultos jóvenes, y con mayor nivel de estudios.

El 93\% de las personas en España piensa que la investigación científica y tecnológica es beneficiosa para hacer frente a las enfermedades y las epidemias, y el $88 \%$ cree que este tipo de investigación permite mejorar su calidad de vida. Además, el $87 \%$ entiende que la investigación científica propicia el desarrollo económico de la sociedad. Los españoles y las españolas opinan que la profesión científica es vocacional y se elige más por motivos personales que por incentivos externos. Consideran que es una profesión que compensa personalmente, aunque tiene poco reconocimiento social y está mal remunerada económicamente.

Sin embargo, ninguna de las dos encuestas mencionadas se plantea el estudio de las posibles diferencias habidas entre las mujeres y los hombres de ciencia en la percepción pública de la profesión científica. Es precisamente la variable "género" la que nos interesa conocer y a la que pretendemos aproximarnos mediante los grupos de discusión.

\section{La notoriedad de las mujeres científicas en Internet.}

Los primeros resultados alcanzados en la Investigación del Proyecto IFIGIC, conciernen al análisis de la información científica en Internet. Se trata de los datos obtenidos sobre las investigadoras y los investigadores, en el estudio retrospectivo de la información científica emitida en Internet, correspondiente a los últimos cinco meses, hasta el día catorce de octubre de 2012. La ficha técnica del estudio incluye los siguientes aspectos:

- Fecha de recogida de datos: De junio a octubre de 2012.

- Búsqueda en los sitios de Internet con información científica en español.

- La base de la información es lo publicado sobre ciencia, investigación y tecnología en los meses citados.

- Las etiquetas de análisis son Investigadoras vs Investigadores.

- El software informático utilizado para el rastreo y análisis de la información ha sido el programa Swotti Premium.

Con este estudio se obtuvo una base documental de 6.735 referencias científicas digitales sobre investigadoras e investigadores. En el análisis se observa un claro predominio de la información sobre la opinión. El ochenta y nueve por ciento del total, es decir 5.998 de las referencias, corresponden a menciones informativas. El once por ciento restante, 737 referencias, lo constituyen opiniones.

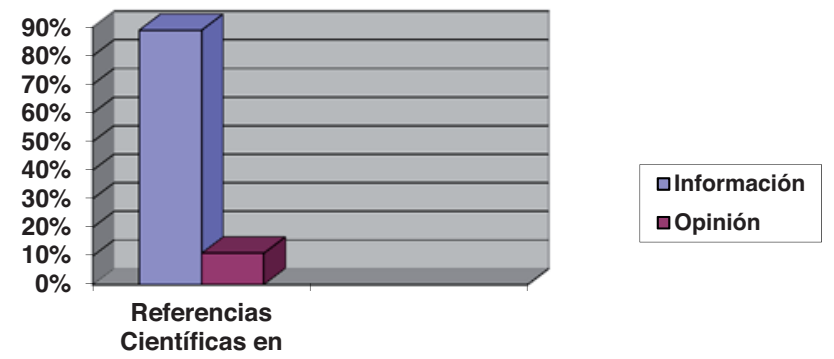

Gráfico 1: Referencias Científicas en Internet: información vs. opinión. 
En el ámbito de la información se aprecia un dato preocupante. Apenas un $12 \%$ de las menciones corresponden a mujeres investigadoras, frente al $88 \%$ de las citas dedicadas a los investigadores. Y estos porcentajes se mantienen prácticamente iguales en el terreno de las opiniones. Sólo el once por ciento se trata de opiniones relativas a las investigadoras.

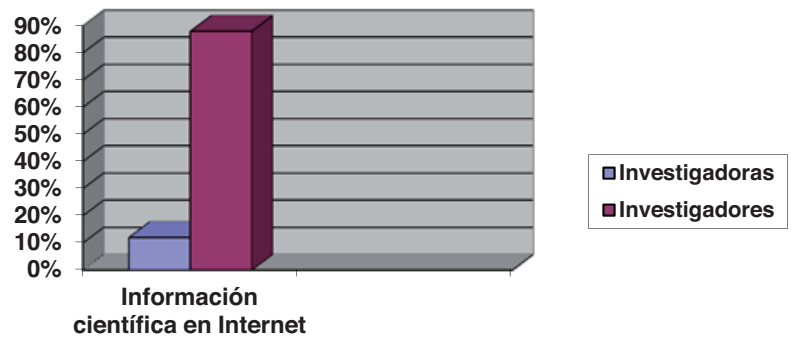

Gráfico 2: Información científica en Internet: investigadores vs. Investigadoras.

Si bien es cierto que la etiqueta "investigadores" puede aludir al plural de las personas dedicadas a la investigación, incluyendo tanto a investigadoras como a investigadores, lo innegable es que la proporción destinada explícitamente a las mujeres científicas, tanto en la información como en la opinión, resulta exigua en términos absolutos.

Por otro lado, las opiniones formuladas en Internet sobre las investigadoras y los investigadores, que representan el once por ciento del total de las referencias, son definitivamente favorables. E1 78\% de las opiniones acerca de las investigadoras, y el $89 \%$ sobre los investigadores, expresan atributos positivos.

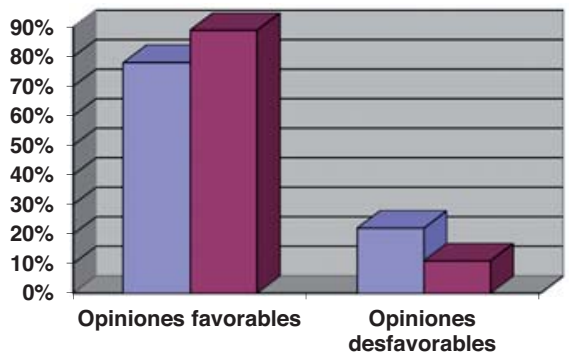

口Investigadoras

口Investigadores

Gráfico 3: Opiniones favorables y desfavorables en Internet: investigadores vs. investigadoras.

En cuanto a las características atribuidas a los investigadores e investigadoras en las informaciones y opiniones digitales, se pueden encontrar algunas diferencias peculiares. Para empezar se comprueba, en lógica correspondencia con el número de referencias, una clara desproporción en el número de calificaciones relativas a los investigadores en relación con las apreciaciones referidas a las investigadoras, que se explicita en una variación de tres a una. Veamos las características más significativas en cada caso. 
Las investigadoras se describen con los siguientes términos: agradecidas, brillantes, de alto nivel, didácticas, dispuestas, distinguidas, eminentes, excepcionales, expertas, ganadoras, grandes, magistrales, más famosas, más importantes, más relevantes, notables, trabajos premiados, sensibles, talento y éxito.

Por su parte, los investigadores se identifican con los siguientes locuciones: adelantado, altamente beneficiosos, brillante, bueno, buenos, calidad, críticos, cualificado, altamente cualificados, conocido, más conocidos, de alto nivel, de primera clase, distinguidos, desarrollados, desconcertado, descubren, descubrimiento, descubrimientos, didáctica, especialistas, especializados, excelentes, éxito, experimentados, experto, expertos, muy expertos, habilidad, ilustres, imparcial, importante, importantes, innovador, innovadores, interesados, interesante, líder, líderes, lógico, intelectual, intelectuales, maestros, mejores, mencionados, modernos, muchos, importantes, muy grande, pionero, positivo, premiado, premiados, premian, preocupados, preparados, prestigioso, prestigiosos, primer nivel, punteros, reclamado, reconocido, reconocidos, relevante relevantes, reputados, sabios, selecto, sensibles, sorprendente, superlativos, talento, talentos, tan famosos, arduo trabajo, único, únicos.

En otro orden de cosas, los soportes en los que se encuentra ubicada la información científica en Internet, son mayoritariamente las webs y los medios digitales. Así se demuestra en el ochenta por ciento de los casos, seguidos a gran distancia de los blogs, en un catorce por ciento de las referencias.

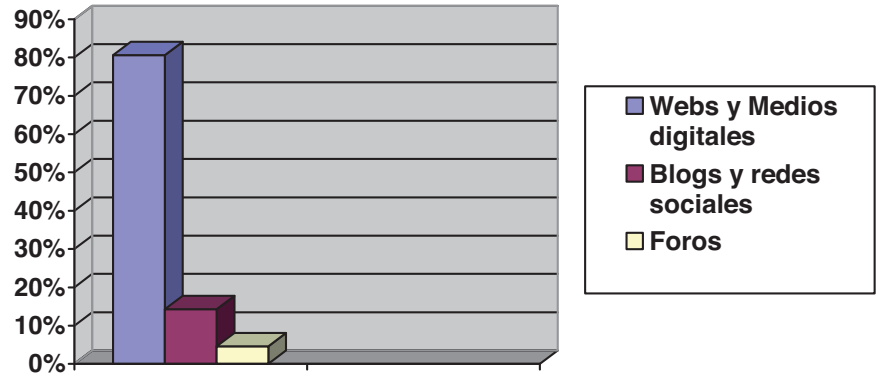

Gráfico 4: Soportes de la información científica en Internet.

El estudio ofrece también interesantes datos sobre la estacionalidad de la información. Se aprecian unos muy bajos niveles informativos, tanto de las investigadoras como sobre los investigadores, durante los meses de mayo, junio, julio y agosto. En los meses siguientes, septiembre y octubre, se produce una mayor concentración de noticias y científicas. No obstante, dado que el porcentaje de referencias a los investigadores es aproximadamente siete veces mayor que el de las investigadoras, parece lógico, como se observa en los resultados del estudio, que el fenómeno de la estacionalidad resulte extraordinariamente más significativo en el caso de las menciones y opiniones relativas a los investigadores. 


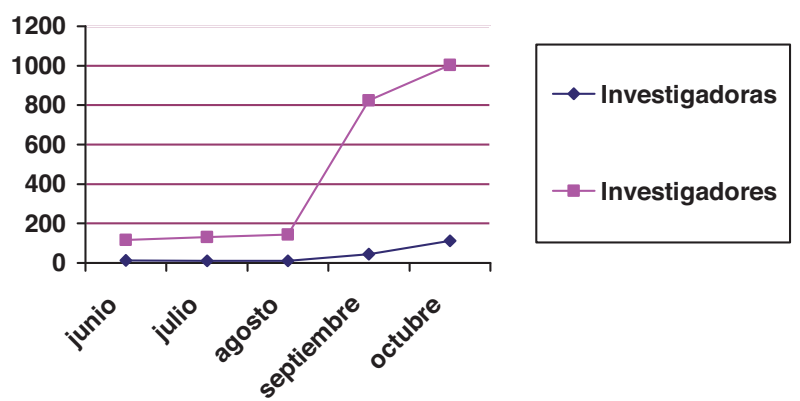

Gráfico 5. Estacionalidad de la información científica en Internet.

Debemos acabar con unas conclusiones precisas y terminantes. Los resultados del estudio digital, antes pormenorizado, no dejan lugar a dudas. Lamentablemente, las mujeres son prácticamente invisibles en los mensajes científicos publicados en Internet. De este modo, se verifica la hipótesis de nuestra investigación en el caso específico de este medio. Ahora, siguiendo el proceso de nuestro Proyecto, tendremos que comprobar cuáles son las circunstancias concretas de las restantes caras del poliedro informativo e imaginario.

\section{Referencias bibliográficas}

AIMC (2012): Resumen General EGM. Abril 2011 a marzo de 2012. Madrid, AIMC. AIMC (2012): Audiencia de Internet EGM. Febrero / marzo de 2012. Madrid, AIMC.

AIMC Y OJD (2012): Informe ejecutivo. Estudio sobre la relación audiencia y difusión en los medios gráficos (lectores por ejemplar). Madrid, AIMC /OJD/PGD. 27 de septiembre de 2012.

BERGER, Peter y LUCHMANN, Thomas (1968): La construcción social de la realidad. Buenos Aires, Amorrortu.

BLUMER, Herbert. (1982): El Interaccionismo simbólico, perspectiva y método. Barcelona, Hora D.L.

FUNDACIÓN BBV (2012): Estudio Internacional de Cultura Científica de la Fundación BBVA. Comprensión de la ciencia. Departamento de Estudios Sociales y Opinión Pública de la Fundación BBVA. Publicado el 8 de mayo de 2012.

FUNDACIÓN ESPAÑOLA PARA LA CIENCIA Y LA TECNOLOGÍA. FECYT (2012): VI Encuesta de Percepción Social de la Ciencia 2012. Madrid, Ministerio de Economía y Competitividad. 24-10-2012.

ENTMAN, Robert M. (1993): "Framing: Toward Clarification of a Fractured Paradigm". En Journal of Communication. Vol 43, nº 4, pp. 51-58.

GOFFMAN, Erving (1974): Frame analysis: An essay on the organization of experience. London: Harper and Row. Ed. en español: Frame analysis: los marcos de la experiencia. Madrid, Centro de Investigaciones Sociológicas, 2006. 
McCOMBS, Maxwell \& SHAW, Donald. L. (1972): "The agenda-setting function of the mass media". Public Opinion Quarterly V. 36 (2), pp. 176-187.

MEAD, George. H. (1968): Espiritu, persona y sociedad. Buenos Aires, Piadós.

OJD (2012): Difusión de medios impresos en http://www.ojd.es/OJD/Portal/diarios_ojd/_4DOSpuiQo1Y_FOivPcLIIA. 26 de julio 2012.

RIGAL, Inés (2012): “¿Quién nos cuenta lo que pasa?” YO DONA. 3 de marzo de 2012, pp. 14-16.

\section{María Teresa GARCÍA NIETO}

Universidad Complutense de Madrid

Facultad de Ciencias de la Información

Departamento de Comunicación Audiovisual y Publicidad II

Profesora Titular de Universidad 